\title{
STABILITY IN PRO-HOMOTOPY THEORY
}

\author{
by R. M. SEYMOUR \\ (Received 30th January 1989)
}

\begin{abstract}
If $\mathscr{C}$ is a category, an object of pro- $\mathscr{C}$ is stable if it is isomorphic in pro- $\mathscr{C}$ to an object of $\mathscr{C}$. A local condition on such a pro-object, called strong-movability, is defined, and it is shown in various contexts that this condition is equivalent to stability. Also considered, in the case $\mathscr{C}$ is a suitable model category, is the stability problem in the homotopy category $\mathrm{Ho}($ pro- $\mathscr{C})$, where pro- $\mathscr{C}$ has the induced closed model category structure defined by Edwards and Hastings [6].
\end{abstract}

1980 Mathematics subject classification (1985 Revision): S5P99.

\section{Introduction}

Let $\mathscr{C}$ be any category and pro- $\mathscr{C}$ the category of pro-objects in $\mathscr{C}([1$, appendix $])$. There is a full embedding of $\mathscr{C}$ in pro- $\mathscr{C}$ obtained by regarding an object of $\mathscr{C}$ as a proobject indexed by the trivial, one point, category. The stability problem in pro- $\mathscr{C}$ is then the following question. When is an object of pro- $\mathscr{C}$ isomorphic in pro- $\mathscr{C}$ to an object of $\mathscr{C}$ ?

Let $\mathbf{S}$ be the category of simplicial sets, and $\mathbf{S}_{0}$ the associated category of pointed, connected objects in $\mathrm{S}$. There is a homotopy category $\mathrm{Ho}\left(\mathbf{S}_{0}\right)$ obtained from the standard closed model category structure on $\mathbf{S}$ (see, for example, [3, §2]). Largely because of its significance for shape theory, the stability problem in pro-Ho(S $\mathbf{S}_{0}$ ) (or equivalently, the pro-category of spaces having the pointed homotopy type of pointed $C W$-complexes) has been extensively studied (e.g. $[4,5,6,9])$. Typical results have given conditions on algebraic invariants associated to pro-homotopy types which imply stability. For example, Edwards and Geoghegan prove:

Theorem 0. ([4], see also [6, Theorem 5.5.5.]). Let $\mathbf{X}$ be an object of pro- $\mathbf{S}_{0}$, and suppose that $\pi_{k}(\mathbf{X})$ is stable in the category of pro-groups for each $k \geqq 1$. Then $\mathbf{X}$ is stable in pro-Ho(S $\left.\mathbf{S}_{0}\right)$ if $\sup _{i}\left\{\operatorname{dim} X_{i}\right\}<\infty$.

We note two extensions that can be made to Theorem 0 . Firstly, if $\mathbf{S} p$ is the category of simplicial spectra, and $S p_{0}$ the associated category of pointed, connected objects in $\mathbf{S} p$, then there is a canonical closed model category structure on $\mathbf{S} p$, yielding a homotopy category $H o\left(\mathbf{S} p_{0}\right)$. Then it is noted in [6, Theorem 5.5.5], that Theorem 0 also holds with $\mathbf{S}_{0}$ replaced by $\mathbf{S} p_{0}$.

Secondly, for any suitably nice closed model category $\mathscr{C}$ (S and $\mathrm{S} p$ are "nice"), Edwards and Hastings define a natural closed model category structure on pro- $\mathscr{C},[6]$. Thus, we obtain a strong pro-homotopy category, $H o($ pro- $\mathscr{C})$. The canonical functor 
$\mathscr{C} \rightarrow H o(\mathscr{C})$, extends to a functor pro- $\mathscr{C} \rightarrow$ pro- $H o(\mathscr{C})$ which factors through $H o($ pro- $\mathscr{C})$. We therefore refer to pro- $\mathrm{Ho}(\mathscr{C})$ as the weak pro-homotopy category. The categories $\mathrm{Ho}$ (pro$\mathscr{C})$ are fruitfully exploited by the above authors in various geometrical contexts $([6, \$ 6$, 7 and 8]). For present purposes, we note that the stability problem may also be studied in $\mathrm{Ho}\left(\right.$ pro- $\left.\mathscr{C}_{0}\right)$; that is, we can ask when an object of $H o\left(\right.$ pro- $\left.\mathscr{C}_{0}\right)$ is isomorphic in $H o$ (pro- $\left.\mathscr{C}_{0}\right)$ to an object of $H o\left(\mathscr{C}_{0}\right)$, (again, $\mathrm{Ho}\left(\mathscr{C}_{0}\right)$ is embedded as a full subcategory of $H o\left(\right.$ pro- $\left.\mathscr{C}_{0}\right)$ in an obvious way). In [4] (see also [6, 5.5.7]), it is shown that the conclusion of Theorem 0 holds in $\mathrm{Ho}\left(\right.$ pro- $\left.\mathrm{S}_{0}\right)$ if $\mathbf{X}$ is a tower (i.e., a pro-object indexed by the natural numbers).

Theorem 0 is proved by showing that the homotopy $\operatorname{limit}$, holim $\mathbf{X}([6, \S 4])$, is finite under the given hypothesis, and then applying a Whitehead theorem in pro- $H o\left(\mathbf{S}_{0}\right)([6$, 5.5.3]), to show that the natural map $\mathbf{h}$ :holim $\mathbf{X} \rightarrow \mathbf{X}$ is an isomorphism in pro- $H o\left(\mathbf{S}_{0}\right)$. Since a strong Whitehead theorem is now available ([11, Theorem A]), the same proof yields immediately (see also Theorem 2.6 below):

Theorem 1. Let $\mathscr{C}=\mathbf{S}$ or $\mathbf{S} p$. Then the conclusion of Theorem 0 holds in Ho(pro- $\left.\mathscr{C}_{0}\right)$.

Theorems 0 and 1 study the stability problem in terms of algebraic invariants of pro-homotopy types. In this paper, we shall approach the question from a different point of view. Thus, we shall consider local conditions on a pro-homotopy type which guarantee stability.

One such local condition which has been widely studied and accepted as a desirable property of pro-homotopy types is the notion of movability (e.g. [6, \$2]). If $\mathscr{C}$ is any category, an object $\mathbf{X}$ in pro- $\mathscr{C}$ is movable, if for each object $i \in I$ (a small, cofiltering index category for $\mathbf{X}$ ), there is a $j \rightarrow i$ such that, for each $k \rightarrow j$ there is a morphism $\theta$ in $\mathscr{C}$ making the following diagram commute in $\mathscr{C}$

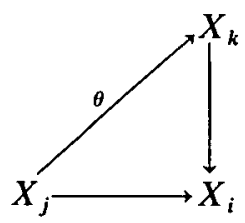

(the unlabelled arrows are bonding maps). However, it is known that a movable object of pro- $\mathrm{Ho}\left(\mathbf{S}_{0}\right)$ need not be stable (e.g., [6, Example 5.5.16]). We must therefore look for something stronger than movability. The basic local condition to be considered in this paper is:

Definition 1.1. An object $\mathbf{X}$ (indexed by $I$ ) in pro- $\mathscr{C}$ is strongly-movable if, for each object $i \in I$, there is a $j \rightarrow i$ such that, for each $k \rightarrow j$, there is an $l \rightarrow k$ and a morphism $\theta$ in $\mathscr{C}$ making the following diagram commute in $\mathscr{C}$

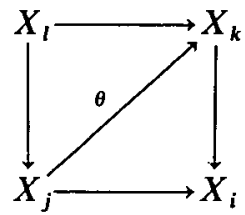

We shall show in various contexts that strong-movability and stability are equivalent. 


\section{Statement of results}

We shall first study the stability problem in pro- $\mathscr{C}$ when $\mathscr{C}$ is sufficiently well behaved. Thus,

Definition 2.1. $\mathscr{C}$ is an EM-category if each morphism $f: A \rightarrow B$ can be factorized in the form $A \stackrel{\vec{f}}{\rightarrow} C \stackrel{u}{\rightarrow} B$, with $\underset{f}{ }$ an epimorphism and $u$ a monomorphism. Further, this factorization is unique in the sense that, if $A \stackrel{f^{\prime}}{\rightarrow} C^{\prime} \stackrel{u^{\prime}}{\rightarrow} B$ is another such, then there is a unique isomorphism $\gamma: C \rightarrow C^{\prime}$ with $\tilde{f}^{\prime}=\gamma \circ f$ and $u=u^{\prime} \circ \gamma$.

Remark 2.2. It is routine to deduce from the uniqueness property that, given a solid arrow diagram

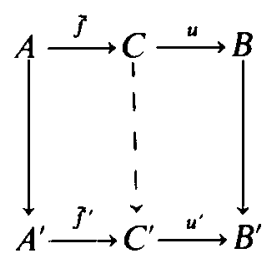

in which the bottom horizontal line is a factorization of $f^{\prime}: A^{\prime} \rightarrow B^{\prime}$, then the dotted arrow exists and is unique.

We can now state the first of our main theorems.

Theorem 2.3. Let $\mathscr{C}$ be an EM-category. Then $\mathrm{X}$ in pro- $\mathscr{C}$ is stable in pro- $\mathscr{C}$ if and only if $\mathbf{X}$ is strongly-movable.

For non EM-categories, more is required. We extend Theorem 2.3 to the general case as follows.

Theorem 2.4. Let $\mathscr{C}$ be any category, and $\mathrm{X}$ an object of pro- $\mathscr{C}$ indexed by a small, cofiltering category $J$. Then $\mathbf{X}$ is stable in pro- $\mathscr{C}$ if and only if $\mathbf{X}$ is strongly-movable, and the functor from $\mathscr{C}$ to Sets given by $K \mapsto \lim _{j \in J} \mathscr{C}\left(K: X_{j}\right)$, is representable (i.e., if $\lim _{j \in J} X_{j}$ exists in $\mathscr{C})$.

We apply Theorem 2.4 to homotopy categories (which, of course, are not EM).

Theorem 2.5. Let $\mathscr{C}$ be a closed model category, and suppose Brown's representability theorem holds in $\mathrm{Ho}\left(\mathscr{C}_{0}\right)$ (i.e., pointed-set-valued homotopy functors on $\mathrm{Ho}\left(\mathscr{C}_{0}\right)$ are representable). Then an object $\mathbf{X}$ in pro- $\mathrm{Ho}\left(\mathscr{C}_{0}\right)$ is stable in pro-Ho( $\left.\mathscr{C}_{0}\right)$ if and only if $\mathbf{X}$ is strongly-movable.

Finally, we extend Theorem 2.5 to obtain a result holding in the strong prohomotopy category. 
Theorem 2.6. Let $\mathscr{C}=\mathbf{S}$ or $\mathbf{S p}$. Then an object $\mathbf{X}$ in pro- $\mathscr{C}_{0}$ is stable in Ho(pro- $\left.\mathscr{C}_{0}\right)$ if and only if it is stable in pro- $\mathrm{Ho}\left(\mathscr{C}_{0}\right)$.

We mention three simple consequences of our theorems. The first is immediate from Theorems 2.4, 2.6 and [8, Corollary 1, p. 227], and the second is immediate from Theorem 2.3 and [6, Theorem C, p. 144].

Proposition 2.7. Under the hypotheses of Theorem 2.4, if $\mathbf{X}$ is dominated by an object $P \in \mathscr{C}$, then $\mathbf{X}$ is stable in pro- $\mathscr{C}$. Under the hypoptheses of Theorem 2.6 , if $\mathbf{X} \in$ pro- $\mathscr{C}_{0}$ is dominated in pro-Ho( $\left.\mathscr{C}_{0}\right)$ by an object $P \in \mathscr{C}_{0}$, then $\mathbf{X}$ is stable in $\mathrm{Ho}\left(\right.$ pro- $\left.\mathscr{C}_{0}\right)$.

Proposition 2.8. Let $\mathbf{G}$ be a strongly-movable pro-group indexed by $J$. Then.

(i) $\lim _{J} \mathbf{G} \rightarrow \mathbf{G}$ is an isomorphism of pro-groups;

(ii) $\lim _{J}^{1} \mathbf{G}=0$;

(iii) $\lim _{J}^{s} \mathbf{G}=0$ for $s>0$ if each $G_{j}$ is abelian.

In [6, 9.3], Edwards and Hastings ask the question: if $\mathbf{f : X} \rightarrow \mathrm{Y}$ is a morphism in $H o\left(\right.$ pro- $\left.\mathbf{S}_{0}\right)$ which is invertible in pro- $H o\left(\mathbf{S}_{0}\right)$, is $\mathbf{f}$ invertible in $H o\left(\right.$ pro- $\left.\mathbf{S}_{0}\right)$ ? The answer to this question is unknown even on the full subcategory tow- $\mathbf{S}_{0}$ generated by the tower objects. However, for towers, Edwards and Hastings prove that, if $\mathrm{f}: \mathbf{X} \rightarrow \mathbf{Y}$ in $\mathrm{Ho}\left(\right.$ tow- $\left.\mathrm{S}_{0}\right)$ is an isomorphism in tow-Ho( $\left.\mathbf{S}_{0}\right)$, then there is some isomorphism $\mathbf{g : X} \rightarrow \mathbf{Y}$ in $H o\left(\right.$ tow- $\left.S_{0}\right)$ whose image in tow-Ho( $\left.\mathbf{S}_{0}\right)$ coincides with that of $f$ [6, Theorem 5.2.9]. But it is not known whether we can take $\mathbf{g}=\mathbf{f}$ in general. The above result suffices to obtain the important corollary that the isomorphism classification of objects is the same in tow- $\mathrm{Ho}\left(\mathrm{S}_{0}\right)$ and $\mathrm{Ho}\left(\right.$ tow- $\left.\mathrm{S}_{0}\right)[6$, Corollary 5.2.17].

Unfortunately, because it is not possible to give a sufficiently rigid construction of $\mathbf{g}$ above, we cannot, with the techniques presently at our disposal, generalize the above result from towers to arbitrary pro-objects. However, using Theorem 2.6 above, we can generalise Corollary 5.2.13 of [6], as follows.

Proposition 2.9. Let $\mathscr{C}=\mathbf{S}$ or $\mathbf{S p}$ and $\mathbf{f}: \mathbf{X} \rightarrow \mathbf{Y}$ be a morphism in $\mathrm{Ho}\left(\right.$ pro- $\left.\mathscr{C}_{0}\right)$ which is an

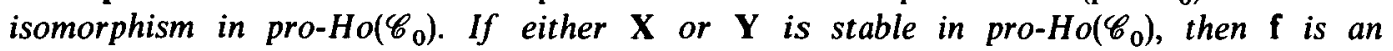
isomorphism in $\mathrm{Ho}\left(\right.$ pro- $\left.\mathscr{C}_{0}\right)$.

We remark that a notion of strong-movability was first introduced by Borsuk [2] in the context of the shape theory of compacta. This was subsequently developed by Mardes[7] and Dydak [3]. For applications to shape theory, see [8, Theorem 8, p. 230, and Remark 2, p. 235]. The abstract definition (1.1) of strong-movability may also be found in [8, p. 226]. Theorem 2.5, with $C$ the category of polyhedra, has been proved by Dydak [3]-see also [8, Theorem 7, p. 228]. Of course, Brown's representability theorem holds for polyhedra, so that Dydak's result follows immediately from ours. However, Theorems 2.4 and 2.5, taken together, bring out what is essential to theorems of this type (unlike the more specific and direct argument given by Dydak), and have much more general applicability. For example, we can take $\mathscr{C}=\mathrm{S} p$, or, more generally, a 
category of diagrams (say, in S) in Theorem 2.5 (these being categories for which a Brown representability theorem is available).

Theorem 2.6 is also proved in a more general form than stated above. In fact, what is required is that $\mathscr{C}$ be "nice" in the sense of Edwards and Hastings [6]; see also [11], so that $\mathrm{Ho}$ (pro- $\mathscr{C}_{0}$ ) exists, and that a Whitehead theorem (see 7.7 below) and a Brown representability theorem hold in $\mathrm{Ho}\left(\mathscr{C}_{0}\right)$. Note also that Theorem 2.6 implies that the shape theory stability results derived from Theorem 2.5 and mentioned earlier, hold in the sense of "strong shape theory", where the strong shape of a space $X$ is defined as the isomorphism class in $H o\left(\right.$ pro- $\left.S_{0}\right)$ of any object of pro-S $S_{0}$ associated to $X$ (e.g., the Vietoris nerve of $X$ ).

The remainder of the paper is arranged as follows. In Section 3, we prove the "only if" assertion of our theorems, and also a special case of Theorem 2.4 (Proposition 3.6). In Section 4 , we prove Theorem 2.3 by reducing it to this special case. Theorem 2.4 is proved in Section 5. The main step here is a "change of limits" theorem (Proposition 5.1) and its Corollaries (5.2 and 5.4), which effectively allow us to reduce Theorem 2.4 to Theorem 2.3. However, owing to its length, the proof of the technical Proposition 5.1 is deferred to Section 8. In Section 6, we apply Brown's representability theorem for homotopy functors to deduce Theorem 2.5. In Section 7, we apply the "induction up from towers' technique" pioneered in [11] to obtain Theorem 2.6; we also prove Proposition 2.9. Finally, in an appendix, we collect together the technical results on proobjects and morphisms which we use.

\section{Strong movability}

We first introduce some convenient notation.

Notation 3.1. Let $\mathbf{X}$ be a strongly-movable object of pro- $\mathscr{C}$ indexed by $I \in$ Cofilt (see Appendix). Given $i \in I$, a morphism $j \rightarrow i$ in $I$ will be called a morphism of strong movability for $i$ (with respect to $\mathbf{X}$ ), if $j \rightarrow i$ satisfies the condition of Definition 1.1. We abbreviate the above terminology to MOSM for $i$.

Clearly, if $\mathbf{X}$ is strongly-movable, then given $i \in l$, there is a MOSM for $i$. The following is obvious from the definitions.

Lemma 3.2. If $i \rightarrow i^{\prime}$ is any morphism in $I$, and $j \rightarrow i$ is a MOSM for $i$ with respect to $\mathbf{X}$, then the composite $j \rightarrow i^{\prime}$ is a MOSM for $i^{\prime}$.

The following proposition is immediate from [8, Theorem 5, p. 227].

Proposition 3.3. Let $\mathbf{X}$ and $\mathbf{Y}$ be objects of pro- $\mathscr{C}$ with $\mathbf{X} \cong \mathbf{Y}$. Then if $\mathbf{Y}$ is strongly-movable, so is $\mathbf{X}$.

We can now easily deduce the "only if" part of our main theorems, namely 
Corollary 3.4. Let $\mathbf{X}$ be an object of pro- $\mathscr{C}$. Then if $\mathbf{X}$ is stable in pro- $\mathscr{C}$, it is strongly-movable.

Proof. Let $\mathrm{X} \cong X$ in pro- $\mathscr{C}$, with $X$ in $\mathscr{C}$. Regarded as a pro-object indexed by the trivial, one point category, $X$ is clearly strongly-movable, and so the corollary follows from Proposition 3.3.

Finally, in this section, we shall prove a special case of Theorem 2.3 (though without the EM assumption).

Lemma 3.5. Let $\mathbf{X}$ be a strongly-movable object of pro- $\mathscr{C}$, and suppose that each bonding morphism $X_{j} \rightarrow X_{i}$ is an epimorphism. Then, given $i$, there is a $j \rightarrow i$ such that, for each $k \rightarrow j$, the induced bonding morphism $X_{k} \rightarrow X_{j}$ is an isomorphism.

Proof. Choose $j \rightarrow i$ to be a MOSM for $i$. Then, given $k \rightarrow j$, we may construct a diagram

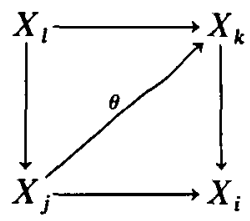

Consider the composite $\theta \circ\left(X_{k} \rightarrow X_{j}\right): X_{k} \rightarrow X_{k}$. We have:

$$
\theta \circ\left(X_{k} \rightarrow X_{j}\right) \circ\left(X_{l} \rightarrow X_{k}\right)=\theta \circ\left(X_{l} \rightarrow X_{j}\right)=\left(X_{l} \rightarrow X_{k}\right)
$$

Since $X_{l} \rightarrow X_{k}$ is an epimorphism, we deduce that $\theta \circ\left(X_{k} \rightarrow X_{j}\right)=$ identity on $X_{k}$. Conversely, consider $\left(X_{k} \rightarrow X_{j}\right) \circ \theta: X_{j} \rightarrow X_{j}$. We have:

$$
\left(X_{k} \rightarrow X_{j}\right) \circ \theta \circ\left(X_{l} \rightarrow X_{j}\right)=\left(X_{k} \rightarrow X_{j}\right) \circ\left(X_{l} \rightarrow X_{k}\right)=\left(X_{l} \rightarrow X_{j}\right)
$$

Again, $X_{l} \rightarrow X_{j}$ an epimorphism implies that $\left(X_{k} \rightarrow X_{j}\right) \circ \theta=$ identity on $X_{j}$. Thus, $X_{k} \rightarrow X_{j}$ is an isomorphism with inverse $\theta$.

Proposition 3.6. Let $\mathrm{X}$ be a strongly-movable object of pro- $\mathscr{C}$ all of whose bonding morphisms $X_{j} \rightarrow X_{i}$ are epimorphisms. Then $\mathbf{X}$ is stable in pro- $\mathscr{C}$.

Proof. Let $\mathrm{X}$ be indexed by $I \in$ Cofilt. Choose any $i_{0} \in I$. By Lemma 3.5, we may choose $j_{0} \rightarrow i_{0}$ such that, for each $j \rightarrow j_{0}$, the induced map $X_{j} \rightarrow X_{j_{0}}$ is an isomorphism.

Let $S\left(j_{0}\right) \subset I$ be the full subcategory of $I$ generated by the successors of $j_{0}$; that is, by those $j \in I$ for which there exists a $j \rightarrow j_{0}$. Then, given $i \in I$, we may construct a diagram, $j_{0} \leftarrow j \rightarrow i$. Thus, $j \in S\left(j_{0}\right)$ and there is a $j \rightarrow i$. It follows that $S\left(j_{0}\right)$ is cofiltering and the inclusion $S\left(j_{0}\right) \subset I$ is cofinal (cf. [11, Proposition 1.1(ii)]). Hence, by A.1, $\mathbf{X}$ is isomorphic 
to the restricted pro-object indexed by $S\left(j_{0}\right)$. But this latter object has all its bonding maps isomorphisms, and so is obviously stable.

\section{Proof of Theorem 2.3}

Throughout this section, $\mathscr{C}$ will be an EM-category (Definition 2.1).

Let $\mathbf{X}$ be a strongly-movable object of pro- $\mathscr{C}$ indexed by $I \in$ Cofilt. Given $i \in I$, let $j \rightarrow i$ be a MOSM for $i$ (Section 3). Decompose the bonding morphism $X_{j} \rightarrow X_{i}$ into a composite $X_{j} \stackrel{\beta_{i}}{\rightarrow} C_{i} \stackrel{\alpha_{i}}{\rightarrow} X_{i}$ with $\beta_{i}$ an epimorphism and $\alpha_{i}$ a monomorphism.

Lemma 4.1. $\quad C_{i}$ is independent (up to unique isomorphism preserving $\alpha_{i}$ ) of the choice of MOSM for $i$.

Proof. Let $j^{\prime} \rightarrow i$ be another MOSM for $i$, and $X_{j^{\prime}} \stackrel{\beta_{i}}{\rightarrow} C_{j}^{\prime a_{i}} \rightarrow X_{i}$ the associated decomposition of $X_{j^{\prime}} \rightarrow X_{i}$. Choose a diagram in $I, j^{\prime} \leftarrow k \rightarrow j$. Since $j \rightarrow i$ and $j^{\prime} \rightarrow i$ are MOSMs for $i$, we may construct diagrams
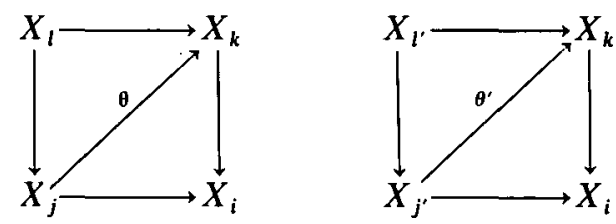

Decompose $X_{k} \rightarrow X_{i}$ as $X_{k} \stackrel{\beta}{\rightarrow} D \stackrel{\alpha}{\rightarrow} X_{i}$ with $\beta$ an epimorphism and $\alpha$ a monomorphism. By uniqueness of such decompositions, it follows that the dotted arrows in the diagrams below exist and are unique (see Remark 2.2)
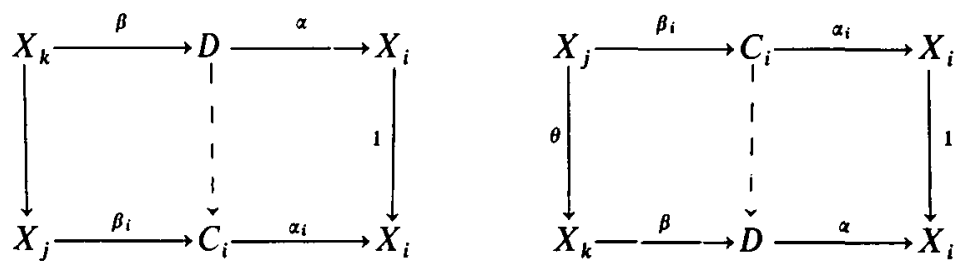

Let $\gamma: D \rightarrow D$ be the composite of the two dotted arrows. Then $\alpha \circ \gamma=1_{X_{i}} \circ \alpha=\alpha \circ 1_{D}$, whence $\gamma=1_{D}$ since $\alpha$ is a monomorphism.

Similarly, the composite $C_{i} \rightarrow D \rightarrow C_{i}$ is the identity on $C_{i}$, and so the unique map $C_{i} \rightarrow D$ is an isomorphism. Again, by the same argument, we have a unique isomorphism $D \rightarrow C_{i}^{\prime}$ satisfying $\alpha_{i} \circ\left(D \rightarrow C_{i}^{\prime}\right)=\alpha$. Thus, the composite $C_{i} \rightarrow D \rightarrow C_{i}^{\prime}$ is a unique isomorphism satisfying $\alpha_{i}=\alpha_{i}^{\prime} \circ\left(C_{i} \rightarrow C_{i}^{\prime}\right)$, which proves the lemma.

For each $i \in I$, choose, once and for all, a MOSM for $i$. This choice then gives a definite assignment $i \mapsto C_{i}$, from the objects of $I$ to the objects of $\mathscr{C}$, and a definite assignment $i \mapsto \alpha_{i}$, from the objects of $I$ to the morphisms of $\mathscr{C}$. 
Lemma 4.2. The assignments $i \mapsto C_{i}$ and $i \mapsto \alpha_{i}$ extend uniquely to a functor $\mathrm{C}: I \rightarrow \mathscr{C}$, and $a$ natural transformation $\alpha: \mathbf{C}, \underset{\sim}{\mathbf{X}}$. Further, all the bonding morphisms of $\mathbf{C}$ are epimorphisms.

Proof. Given $i \rightarrow i^{\prime}$ in $I$, let $j \rightarrow i$ and $j^{\prime} \rightarrow i^{\prime}$ be the chosen MOSMs for $i$ and $i^{\prime}$ which define $C_{i}, C_{i^{\prime}}, \alpha_{i}$ and $\alpha_{i^{\prime}}$. By Lemma 3.2, the composite $j \rightarrow i \rightarrow i^{\prime}$ is also a MOSM for $i^{\prime}$. If the bonding map $X_{j} \rightarrow X_{i^{\prime}}$ decomposes as $X_{j} \stackrel{\bar{\beta}_{i} \bar{C}_{i^{\prime}}}{\bar{\alpha}_{i^{\prime}}} X_{i^{\prime}}$, then it follows from Lemma 4.1 that there is a unique isomorphism $\bar{C}_{i^{\prime}} \cong C_{i^{\prime}}$ satisfying $\alpha_{i^{\prime}} \circ\left(\bar{C}_{i^{\prime}} \rightarrow C_{i^{\prime}}\right)=\bar{\alpha}_{i^{\prime}}$.

Now, by Remark 2.2, the dotted arrow in the following diagram exists and is unique.

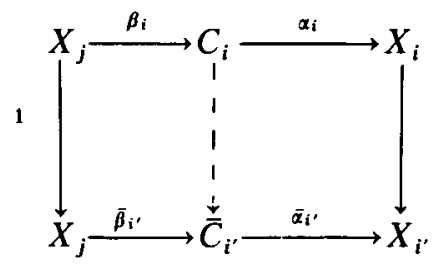

Hence, there is a unique morphism $C_{i} \rightarrow \bar{C}_{i^{\prime}} \cong C_{i^{\prime}}$ satisfying $\alpha_{i^{\prime}} \circ\left(C_{i} \rightarrow C_{i^{\prime}}\right)=\left(X_{i} \rightarrow X_{i^{\prime}}\right) \circ \alpha_{i}$. Functoriality now follows by uniqueness.

Finally, since $\left(C_{i} \rightarrow \bar{C}_{i^{\prime}}\right) \circ \beta_{i}=\bar{\beta}_{i^{\prime}}$, and $\bar{\beta}_{i^{\prime}}$ is an epimorphism, it follows that $C_{i} \rightarrow \bar{C}_{i^{\prime}}$ is an epimorphism. Hence, so is $C_{i} \rightarrow C_{i^{\prime}}$.

Lemma 4.3. $\alpha: \mathrm{C} \rightarrow \mathrm{X}$ is an isomorphism in pro- $\mathscr{C}$.

Proof. Given $i \in I$, let $j \rightarrow i$ be the MOSM for $i$ which defines $C_{i}$ and $\alpha_{i}$. Consider the diagram

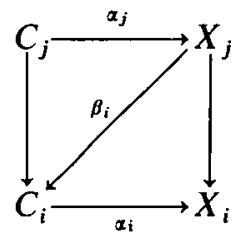

The lower right triangle commutes by definition of $C_{i}, \alpha_{i}$ and $\beta_{i}$. Now, the bonding map $C_{j} \rightarrow C_{i}$ is uniquely characterized by the property, $\alpha_{i} \circ\left(C_{j} \rightarrow C_{i}\right)=\left(X_{j} \rightarrow X_{i}\right) \circ \alpha_{j}$ (because $\alpha_{i}$ is a monomorphism). We therefore have,

$$
\alpha_{i} \circ\left(C_{j} \rightarrow C_{i}\right)=\left(X_{j} \rightarrow X_{i}\right) \circ \alpha_{j}=\alpha_{i} \circ \beta_{i} \circ \alpha_{j}
$$

and so by uniqueness we must have $\left(C_{j} \rightarrow C_{i}\right)=\beta_{i} \circ \alpha_{j}$, showing that the upper left triangle also commutes. That $\alpha: \mathbf{C} \rightarrow \mathbf{X}$ is an isomorphism in pro- $\mathscr{C}$ now follows from Proposition A.3. 
4.4. Proof of Theorem 2.3. If $X$ in pro- $\mathscr{C}$ is stable, then $X$ is strongly-movable by Corollary 3.4. Conversely, if $\mathscr{C}$ is EM and $X$ is strongly-movable, then $\mathbf{X} \cong \mathbf{C}$ by Lemma 4.3. Hence, $\mathbf{C}$ is strongly-movable by Proposition 3.3. But $\mathbf{C}$ has all its bonding maps epimorphisms (Lemma 4.2), and so is stable by Proposition 3.6. It now follows that $\mathbf{X}$ is stable, as required.

\section{Interchange of limits and Theorem 2.4}

Most of this section is taken up with change of limit theorems. At the end of the section we shall use these theorems to deduce Theorem 2.4 .

Our central proposition is the following: the proof will be given in Section 8 .

Proposition 5.1. Let $I$ be any small category and $J \in$ Cofilt. Let $\mathrm{F}: I \times J \rightarrow \mathscr{C}$ be a bifunctor, and $\hat{\mathbf{F}}: J \rightarrow \mathscr{C}^{I}$ the "adjoint" of $\mathbf{F}$. Regard $\hat{\mathbf{F}}$ as an object of pro- $\mathscr{C}^{I}$. Then, if $\hat{\mathbf{F}}$ is stable in pro- $\mathscr{C}^{I}$, the canonical change of limits map

$$
\kappa: \operatorname{colim} \lim _{j \in J} F_{i j} \rightarrow \underset{j \in J}{\lim } \operatorname{colim} F_{i j}
$$

is an isomorphism in $\mathscr{C}$ (provided the limits and colimits exist).

Now let $\mathscr{C}$ be an EM-category. If $\gamma: F \rightarrow G$ is a morphism in $\mathscr{C}^{I}$, then we may decompose each $\gamma_{i}: F_{i} \rightarrow G_{i}$ uniquely in the form $F_{i} \stackrel{\mathcal{F}_{i}}{\rightarrow} C_{i} \stackrel{u_{i}}{\rightarrow} G_{i}$, with $\tilde{\gamma}_{i}$ an epimorphism and $u_{i}$ a monomorphism. By Remark 1.2, if $i \rightarrow i^{\prime}$ is a morphism in $I$, then there is a unique morphism $C_{i} \rightarrow C_{i}$, making the following diagram commute

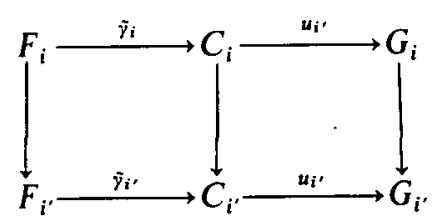

It follows that $i \mapsto C_{i}$ defines an object $C$ in $\mathscr{C}^{I}$, and that the $u_{i}$ and $\tilde{\gamma}_{i}$ define morphisms in $\mathscr{C}^{I}, F \stackrel{\tilde{\Sigma}}{\rightarrow} C \stackrel{u}{\rightarrow} G$, with $u \circ \tilde{\gamma}=\gamma$. Further, each $\tilde{\gamma}_{i}$ an epimorphism and each $u_{i}$ a monomorphism implies that $\tilde{\gamma}$ is an epimorphism and $u$ is a monomorphism in $\mathscr{C}^{I}$. The uniqueness of the above decomposition of $\gamma$ in $\mathscr{C}^{I}$, follows from that in $\mathscr{C}$. Thus, if $\mathscr{C}$ is $\mathrm{EM}$, so is $\mathscr{C}^{I}$.

Corollary 5.2. Let $\mathscr{C}$ be EM. Let $I$ be a small category and $J \in C o f i l t$, and F:I $\times J \rightarrow \mathscr{C}$ a functor. Then, if $\hat{\mathbf{F}}$ is strongly-movable in $\mathscr{C}^{J}$, the canonical map

$$
\kappa: \operatorname{colim} \lim _{i \in I} F_{i j} \rightarrow \lim _{j \in J} \operatorname{colim} F_{i \in I}
$$

is an isomorphism in $\mathscr{C}$. 
Proof. By the above remarks, $\mathscr{C}^{I}$ is EM. Hence, by Theorem $2.3, \hat{\mathbf{F}}$ is stable in $\mathscr{C}^{I}$, and so the corollary follows from Proposition 5.1.

Now let $\mathscr{C}$ be any category, not necessarily EM. Let $\mathbf{X}$ and $\mathbf{K}$ be objects of pro- $\mathscr{C}$ indexed by $J$ and $I$, respectively. Define a functor

$$
\mathbf{F}: I^{o p} \times J \rightarrow \text { Sets }
$$

by $F_{i j}=\mathscr{C}\left(K_{i} ; X_{j}\right)$.

Lemma 5.3. If $\mathbf{X}$ is strongly-movable in pro- $\mathscr{C}$, then $\hat{\mathbf{F}}$ is strongly-movable in pro-(Sets) ${ }^{\text {Iop }}$.

Proof. We have $\hat{F}_{j}=\mathscr{C}\left(K ; X_{j}\right)$ for $j \in J$. Given $j \in J$, let $k \rightarrow j$ be a MOSM for $j$ with respect to $X$. Then, given $k^{\prime} \rightarrow k$, we may find $l \rightarrow k^{\prime}$ and a $\theta$ such that the following diagram commutes

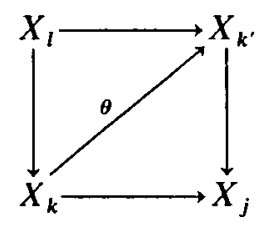

This yields a diagram in $(\text { Sets })^{\text {Iop }}$

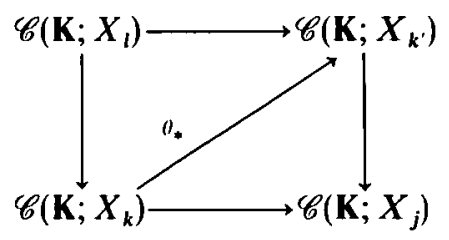

Thus, $\theta_{*}: \hat{F}_{k} \rightarrow \hat{F}_{k^{\prime}}$ exhibits $k \rightarrow j$ as a MOSM for $j$ with respect to the pro-object $\hat{\mathbf{F}}$.

Corollary 5.4. Let $\mathscr{C}$ be any category and $\mathrm{X}$ and $\mathrm{K}$ in pro- $\mathscr{C}$ be indexed by $J$ and $I$, respectively. Then, if $\mathbf{X}$ is strongly-movable in pro- $\mathscr{C}$, the canonical map

$$
\kappa: \operatorname{colim} \lim _{i \in I} \mathscr{C}\left(K_{i} ; X_{j}\right) \rightarrow \underset{j \in J}{\lim } \operatorname{colim} \mathscr{C} \mathscr{C}\left(K_{i} ; X_{j}\right)=\operatorname{pro}-\mathscr{C}(\mathbf{K} ; \mathbf{X})
$$

is an isomorphism of sets. 
Proof. Sets is EM and admits all small limits and colimits. The corollary now follows from Lemma 5.3 and Corollary 5.2.

5.5. Proof of Theorem 2.4 First suppose that $X$ is stable in pro- $\mathscr{C}$. Then $X$ is strongly-movable by Corollary 3.4. Let $\mathbf{h}: X \rightarrow \mathbf{X}$ be an isomorphism in pro- $\mathscr{C}$ with $X$ in $\mathscr{C}$. Then, for any $\mathbf{K}$ in pro- $\mathscr{C}, \mathbf{h}_{*}: \operatorname{pro}-\mathscr{C}(\mathbf{K} ; X) \rightarrow$ pro- $\mathscr{C}(\mathbf{K} ; \mathbf{X})$ is an isomorphism. Taking $\mathbf{K}=K$ in $\mathscr{C}$, this reduces to

$$
\mathbf{h}_{*} ; \mathscr{C}(K ; X) \cong \text { pro- } \mathscr{C}(K ; \mathbf{X})=\lim _{J} \mathscr{C}(K ; \mathbf{X})
$$

which shows that the functor $K \mapsto \lim _{J} \mathscr{C}(K ; \mathbf{X})$ is representable on $\mathscr{C}$.

Conversely, suppose that $\mathbf{X}$ is strongly-movable and that $X$ in $\mathscr{C}$ represents the functor $K \mapsto \lim _{J} \mathscr{C}(K ; \mathbf{X})$. Thus, there is an isomorphism, $\mathscr{C}(K ; X) \cong \lim _{j \in J} \mathscr{C}\left(K ; X_{j}\right)=$ pro- $\mathscr{C}(K ; \mathbf{X})$. Taking $K=X$ gives a canonical morphism in pro- $\mathscr{C}, \mathbf{h}: X \rightarrow \mathbf{X}$. We shall show that $\mathbf{h}$ is an isomorphism, and hence that $\mathbf{X}$ is stable.

Let $\mathbf{K}$ be any object of pro- $\mathscr{C}$ indexed by $I$. Then we have natural isomorphisms of sets induced by $\mathbf{h}$

$$
\phi_{i}: \mathscr{C}\left(K_{i} ; X\right) \stackrel{\cong}{\operatorname{gim}} \mathscr{C}\left(K_{i} ; X_{j}\right)
$$

These yield an isomorphism

$$
\phi=\operatorname{colim}_{i \in I} \phi_{i}: \operatorname{colim} \mathscr{C}\left(K_{i \in I} ; X\right) \cong \underset{i \in I}{\cong} \operatorname{colim} \lim _{j \in J} \mathscr{C}\left(K_{i} ; X_{j}\right) .
$$

But, colim ${ }_{i \in I} \mathscr{C}\left(K_{i} ; X\right)=$ pro- $\mathscr{C}(\mathbf{K} ; X)$, and by Corollary 5.4 ,

$$
\kappa: \operatorname{colim} \lim _{i \in I} \mathscr{C}\left(K_{\mathrm{i}} ; X_{j}\right) \rightarrow \lim _{j \in J} \operatorname{colim} \mathscr{C}\left(K_{i} ; X_{j}\right)=\operatorname{pro}-\mathscr{C}(\mathbf{K} ; \mathbf{X})
$$

is an isomorphism. Hence, we have a natural isomorphism

$$
\kappa \circ \phi: \operatorname{pro-} \mathscr{C}(\mathbf{K} ; X) \cong \operatorname{pro-} \mathscr{C}(\mathbf{K} ; \mathbf{X})
$$

which, by construction and naturality is just $\mathbf{h}_{\boldsymbol{*}}$. It now follows formally that $\mathbf{h}$ is an isomorphism in pro- $\mathscr{C}$.

Remark 5.6. If $\mathscr{C}$ is a pointed category (i.e., it has an initial-terminal object ${ }^{*}$ ), then the functor $K \mapsto \lim _{j \in J} \mathscr{C}\left(K ; X_{j}\right)$ takes values in $S_{e t} s_{0}$, the category of pointed sets. By the same proof, we than obtain a pointed version of Theorem 2.4.

\section{Homotopy categories and Theorem 2.5}

Let $\mathscr{C}$ be a closed model category, and $H o(\mathscr{C})$ its associated homotopy category, [10]. Let $\mathscr{C}_{0}$ be the associated category of pointed, connected objects in $\mathscr{C}$. 
A homotopy functor on $\mathscr{C}_{0}$ is a contravariant functor $H: H o\left(\mathscr{C}_{0}\right) \rightarrow \operatorname{Set} s_{0}$, satisfying the following axioms:

6.1 (i) If $\vee$ denotes the coproduct in $\mathscr{C}_{0}$, then for any small indexing set $A$, the canonical map

$$
u: H\left(\bigvee_{\alpha \in A} K_{\alpha}\right) \rightarrow \prod_{a \in A} H\left(K_{\alpha}\right)
$$

is an isomorphism of based sets.

(ii) Given a pushout diagram in $H o\left(\mathscr{C}_{0}\right)$

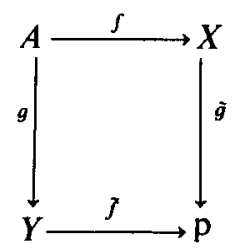

the induced diagram of based sets

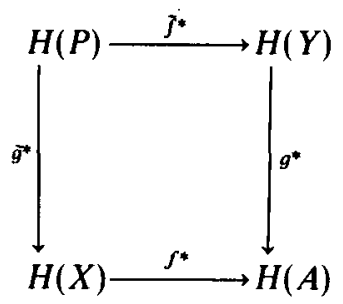

has the Mayer-Vieteris property; i.e., given $x \in H(X), y \in H(Y)$ such that $f^{*}(x)=g^{*}(y)$, then there exists $z \in H(P)$ such that $\tilde{g}^{*}(z)=x$ and $\tilde{f}^{*}(z)=y$.

We shall assume that Brown's Representability Theorem holds for homotopy functors on $\mathscr{C}_{0}$; i.e.

6.2. Brown's Representability Theorem If $H$ is a homotopy functor on $\mathscr{C}_{0}$, then $H$ is representable; i.e., there is an object $X$ in $\mathscr{C}_{0}$ and a natural isomorphism of based-setvalued functors on $\mathrm{Ho}\left(\mathscr{C}_{0}\right)$

$$
T: H o\left(\mathscr{C}_{0}\right)(\ldots, X) \cong H\left(\_\right)
$$

The main step in our proof of Theorem 2.5 is the following.

Lemma 6.3. Let $\mathscr{C}_{0}$ be as above, and suppose that $\mathrm{X}$ is a strongly-movable object of

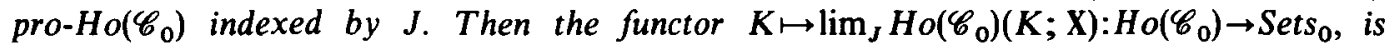
representable. 
Proof. By Brown's Representability Theorem, it suffices to show that $K \mapsto \lim _{J} H o\left(\mathscr{C}_{0}\right)(K ; \mathbf{X})$ is a homotopy functor.

(i) We have

$$
\lim _{J} H o\left(\mathscr{C}_{0}\right)\left(\bigvee_{\alpha} K_{\alpha} ; \mathbf{X}\right) \rightarrow \lim _{J} \prod_{a} H o\left(\mathscr{C}_{0}\right)\left(K_{a} ; \mathbf{X}\right)
$$

is an isomorphism since $K \mapsto H o\left(\mathscr{C}_{0}\right)\left(K ; X_{j}\right)$ is a homotopy functor for each $j \in J$. Axiom 6.1(i) now follows from the fact that limits commute.

(ii) Let $\Delta$ be a finite category and $D: \Delta \rightarrow H o\left(\mathscr{C}_{0}\right)$ a $\Delta$-diagram in $H o\left(\mathscr{C}_{0}\right)$. For $Y$ in

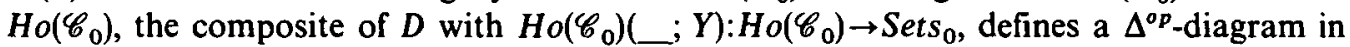
Sets $_{0}$, which we denote by $H o\left(\mathscr{C}_{0}\right)(D ; Y)$. Thus, $j \mapsto H o\left(\mathscr{C}_{0}\right)\left(D ; X_{j}\right)$ defines a pro-object in the category of $\Delta^{o p}$-diagrams in Sets $s_{0}$; i.e., an object of pro- $\left(\operatorname{Set}_{0_{0}}\right)^{\Delta^{o D}}$. Further, by Lemma $5.3, \mathrm{X}$ strongly-movable in pro- $H o\left(\mathscr{C}_{0}\right)$ implies that $H o\left(\mathscr{C}_{0}\right)(D ; \mathbf{X})$ is stronglymovable in pro-(Sets $)^{\Delta^{o p}}$. Thus, since $\operatorname{Sets}_{0}$, and hence $\left(\operatorname{Sets}_{0}\right)^{\Delta^{o p}}$ (see the remarks preceding Corollary 5.2), is EM, it follows from Theorem 2.3 that $H o\left(\mathscr{C}_{0}\right)(D ; \mathbf{X})$ is stable in pro- $(\text { Sets })^{)^{o p p}}$. Hence, the natural map

$$
h: \lim _{j \in J} H o\left(\mathscr{C}_{0}\right)\left(D ; X_{j}\right) \rightarrow H o\left(\mathscr{C}_{0}\right)(D ; \mathbf{X})
$$

is an isomorphism in pro-( $\left.\operatorname{Sets}_{0}\right)^{\Delta^{o p}}$. It follows that, given $j \in J$, there is a $k \rightarrow j$ and a morphism $\theta$ in $\left(\operatorname{Sets}_{0}\right)^{\Delta o p}$ such that the following diagram commutes.

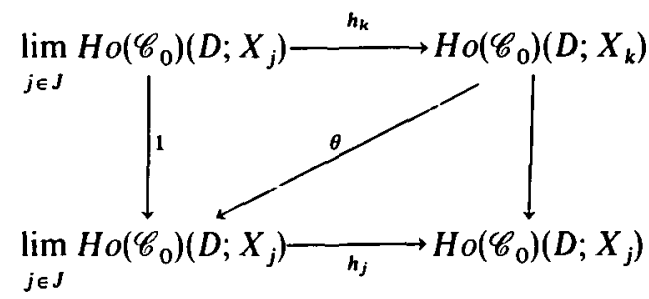

Now take $\Delta$ to be the diagram type

$$
\Delta=\left\{\left.\right|_{3 \bullet \longrightarrow 4} ^{1 \bullet \longrightarrow 2}\right\}
$$

and $D$ to be the $\Delta$-diagram of Axiom 6.1(ii). Then we obtain a commutative diagram in $H o\left(\mathscr{C}_{0}\right)$ from (6.4.)-see overleaf. Here, the $\theta$ 's split the projections $h_{k}$; i.e., $\theta_{i} \circ h_{k}=$ identity, $1 \leqq i \leqq 4$.

Now, suppose given $x \in \lim _{j \in J} H o\left(\mathscr{C}_{0}\right)\left(X ; X_{j}\right)$ and $y \in \lim _{j \in J} H o\left(\mathscr{C}_{0}\right)\left(Y ; X_{j}\right)$ which have the same image in $\lim _{j \in J} H o\left(\mathscr{C}_{0}\right)\left(A ; X_{j}\right)$. Then $h_{k}(x) \in H o\left(\mathscr{C}_{0}\right)\left(X ; X_{k}\right)$ and $h_{k}(y) \in H o\left(\mathscr{C}_{0}\right)\left(Y ; X_{k}\right)$ have the same image in $H o\left(\mathscr{C}_{0}\right)\left(A ; X_{k}\right)$. Thus, since $H o\left(\mathscr{C}_{0}\right)\left(\ldots ; X_{k}\right)$ is a homotopy functor, and hence satisfies Axiom $6.1(\mathrm{ii})$, there is a $\tilde{z} \in H o\left(\mathscr{C}_{0}\right)\left(P ; X_{k}\right)$ 


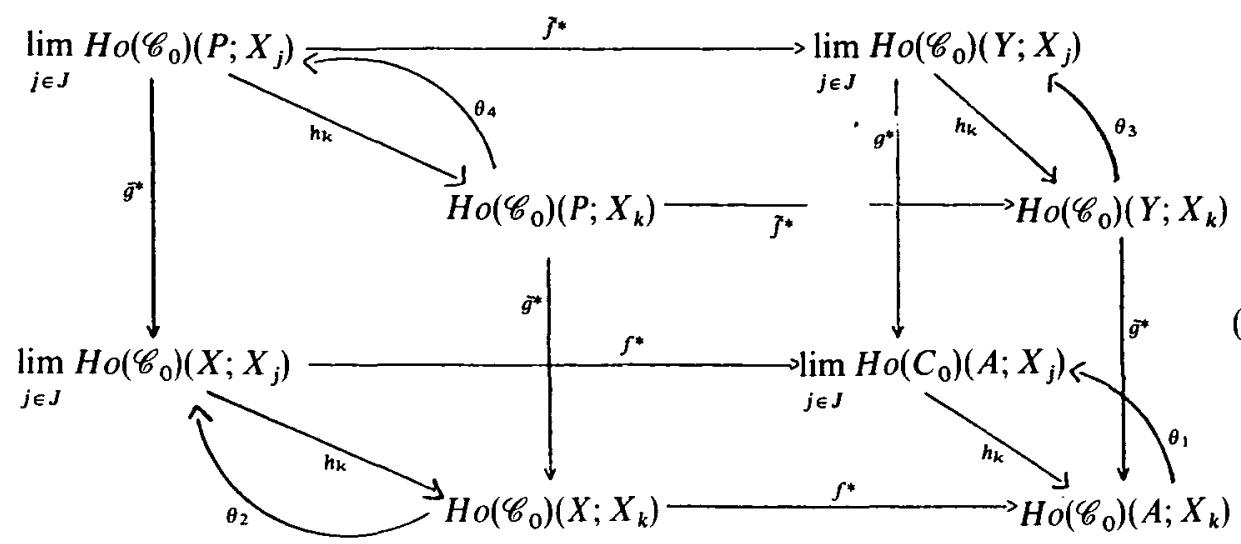

satisfying $\tilde{g}^{*}(\tilde{z})=h_{k}(x)$ and $\tilde{f}^{*}(\tilde{z})=h_{k}(y)$. Take $z=\theta_{4}(\tilde{z}) \in \lim _{j \in J} H o\left(\mathscr{C}_{0}\right)\left(P ; X_{j}\right)$. Then the commutativity of the diagram 6.5 together with the fact that $\theta_{i} \circ h_{k}=$ identity, implies that $\tilde{g}^{*}(z)=x$ and $\tilde{f}^{*}(z)=y$. This shows that $\lim _{j \in J} H o\left(\mathscr{C}_{0}\right)\left(\ldots ; X_{j}\right)$ satisfies Axiom 6.1(ii), as required.

6.6. Proof of Theorem 2.5. If $\mathbf{X}$ is stable, then $\mathbf{X}$ is strongly-movable by Corollary 3.4. Conversely, suppose that $\mathbf{X}$ is strongly-movable. Then $\mathbf{X}$ is stable by Lemma 6.3, Theorem 2.4 and Remark 5.6.

\section{Strong homotopy categories and Theorem $\mathbf{2 . 6}$}

We shall deduce Theorem 2.6 from Theorem 2.5 using the induction technique developed in [11]. For the reader's convenience we begin by reviewing this technique.

Let $\mathscr{C}$ be a nice closed model category ([6, Section 2]). $\mathscr{C}=\mathbf{S}$ or $\mathscr{C}=\mathbf{S} p$ will do. Let $\mathbf{f}: \mathbf{X} \rightarrow \mathbf{Y}$ be a morphism in $\mathscr{C}^{I}$ for some $I \in C S D S$ (see appendix). We wish to give conditions under which $\mathbf{f}$ is an isomorphism in $H o$ (pro- $\left.\mathscr{C}_{0}\right)$.

Let tow- $\mathscr{C}_{0}$ be the full subcategory of pro- $\mathscr{C}_{0}$ generated by the tower objects (i.e., the pro-objects indexed by the natural numbers $\mathbb{N}$ ). The induction technique is based on the following idea. Suppose that $P$ is some property of morphisms in pro- $\mathscr{C}_{0}$ for which we are able to show that any morphism in tow- $\mathscr{C}_{0}$ which satisfies $P$ is an isomorphism in $H o\left(\right.$ pro- $\left.\mathscr{C}_{0}\right)$. Then, if $\mathrm{f}$ is a morphism in pro- $\mathscr{C}_{0}$ which satisfies $P$, under what circumstances can we conclude that $\mathbf{f}$ is an isomorphism in $H o\left(\right.$ pro- $\left.\mathscr{C}_{0}\right)$ ? Thus, we are asking when we can "induct up" from a known result for towers to the same result in the general case.

To state what is required, we introduce the category $\tilde{I}$ of towers in $I([11,1.3])$. The objects of $\tilde{I}$ are towers in $I, \mathbf{i}=\left(i_{0} \leftarrow i_{1} \leftarrow \cdots \leftarrow i_{n} \leftarrow \cdots\right)$, and morphisms in $\tilde{I}$ are level preserving ladder diagrams. There is an evaluation functor $e: \mathbb{N} \times \tilde{I} \rightarrow I$, given on objects by $e(n, \mathbf{i})=i_{n}$. Then $\tilde{I} \in$ Cofilt and $e$ is cofinal ([11, Proposition 1.4]). 
For each $\mathbf{i} \in \tilde{I}$, define a morphism $\mathbf{f}_{\mathrm{j}}: \mathbf{X}_{\mathrm{i}} \rightarrow \mathbf{Y}_{\mathbf{i}}$ in $\mathscr{C}_{0}^{\mathrm{N}}$, by $\left(\mathbf{f}_{\mathrm{i}}\right)_{n}=f_{i_{n}}: X_{i_{n}} \rightarrow Y_{i_{n}}, n \geqq 0$. Let $\tilde{I}(\mathbf{f}) \subseteq \tilde{I}$ be the full subcategory of $\tilde{I}$ generated by those $i \in \tilde{I}$ for which $f_{i}$ is an isomorphism in $H o\left(\right.$ pro- $\left.\mathscr{C}_{0}\right)$. Then our induction technique is based on the following Induction Theorem.

Theorem 7.1. ([11, Theorem 3.1]). If $\tilde{I}(f) \subseteq \tilde{I}$ is cofinal, then $\mathbf{f}$ is an isomorphism in $H o\left(\right.$ pro- $\left.\mathscr{C}_{0}\right)$.

Remarks 7.2. (i) For $\mathbf{i} \in \tilde{I}$, define $e_{i}: \mathbb{N} \rightarrow I$ by $e_{i}(n)=i_{n}$. Then observe that $f_{i}=$ $e_{i}^{*}(\mathbf{f}): e_{i}^{*}(\mathbf{X}) \rightarrow e_{i}^{*}(\mathbf{Y})$.

(ii) Suppose $\mathbf{X}=X$ is an object of $\mathscr{C}$. We may regard $X$ as an object of $\mathscr{C}^{I}$ having all bonding maps the identity on $X$. Then $\mathrm{f}: X \rightarrow \mathbf{Y}$ consists of a collection of morphisms $f_{i}: X \rightarrow Y_{i}$ satisfying $\left(Y_{i} \rightarrow Y_{j}\right) \circ f_{i}=f_{j}$, for each $i \rightarrow j$ in $l$. In this case, for $\mathbf{i} \in \tilde{I}, \mathbf{f}_{\mathbf{i}}: X \rightarrow Y_{\mathbf{i}}=$ $e_{i}^{*}(\mathbf{Y})$ is just the composite $X \stackrel{f}{\rightarrow} \mathbf{Y} \stackrel{\mu_{i}}{\rightarrow} Y_{i}$, where $\mu_{\mathrm{i}}$ is the canonical morphism in pro- $\mathscr{C}_{0}$ given by A.1.

With these preliminaries at hand, we can now take the main step in the proof of Theorem 2.6.

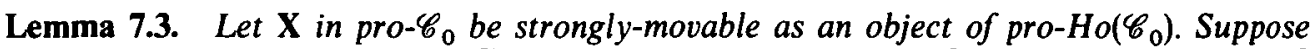
$\mathbf{X}$ is indexed by $I \in C S D S$, and let $\tilde{I}(\mathbf{X})$ be the full subcategory of $\tilde{I}$ generated by those $\mathbf{i} \in \tilde{I}$ for which the canonical morphism $\mu_{i} ; \mathbf{X} \rightarrow e_{i}^{*}(\mathbf{X})=\mathbf{X}_{\mathbf{i}}$, is an isomorphism in pro-Ho( $\left.\mathscr{C}_{0}\right)$. Then the inclusion $\tilde{I}(\mathbf{X}) \subseteq \tilde{I}$ is cofinal.

Proof. Given $\mathbf{i} \in \tilde{I}$, it suffices to construct a morphism $\mathbf{j} \rightarrow \mathbf{i}$ in $\tilde{I}$ with $\mathbf{j} \in \tilde{I}(\mathbf{X})$ (cf. [11, Lemma 1.1(ii)]).

We first show that $\mathbf{j} \rightarrow \mathbf{i}$ can be chosen so that each $j_{n} \rightarrow j_{n-1}$ is a MOSM for $j_{n-1}$ (with respect to $\mathbf{X}$ ). Take $j_{0}=i_{0}$. Suppose that $j_{0} \leftarrow j_{1} \leftarrow \cdots \leftarrow j_{n-1}$, and compatible morphisms $j_{r} \rightarrow i_{r}, 0 \leqq r \leqq n-1$, have been constructed. Choose $j_{n-1} \leftarrow j_{n}^{\prime} \rightarrow i_{n}$ so that the following square commutes

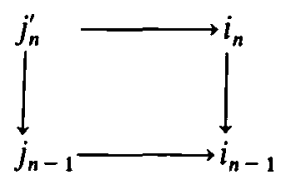

Now take $j_{n} \rightarrow j_{n}^{\prime}$ to be a MOSM for $j_{n}^{\prime}$ (such exists since $\mathbf{X}$ is strongly-movable). Then, by Lemma 3.2, $j_{n} \rightarrow j_{n-1}$ is a MOSM for $j_{n-1}$. Thus, $\mathbf{j} \rightarrow \mathbf{i}$ can be constructed inductively with the required property.

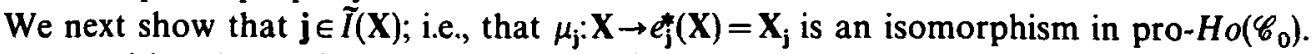
By Proposition A.5 and Remarks 7.2, it suffices to show that, for each morphism $i \rightarrow j_{n}$ in $I$, there is an $m \geqq n$, morphisms $i^{\prime} \rightarrow i$ and $i^{\prime} \rightarrow j_{m}$ in $I$, and $\theta$ in $H o\left(\mathscr{C}_{0}\right)$ such that the following diagram commutes 


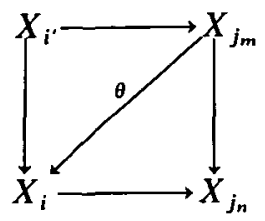

Take $m=n+1$. Choose morphisms in $I, j_{n+1} \leftarrow k \rightarrow i$. Then, since $j_{n+1} \rightarrow j_{n}$ is a MOSM for $j_{n}$ (by construction of $\mathbf{j}$ ), there is an $i^{\prime} \rightarrow k$ and a morphism $\theta^{\prime}$ in $H o\left(\mathscr{C}_{0}\right)$ such that the following diagram commutes

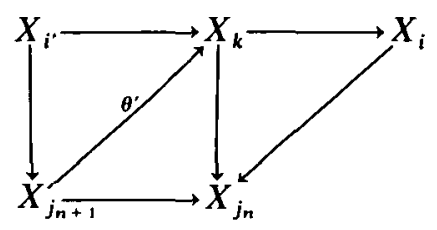

(note: the right-hand triangle commutes since $I \in C S D S$ ). Take $\theta=\left(X_{k} \rightarrow X_{i}\right) \circ \theta^{\prime}$, then we obtain the required diagram 7.4.

Recall that, in $[6, \S 4]$, there is defined a homotopy limit functor.

$$
\text { holim: } H o\left(\text { pro- } \mathscr{C}_{0}\right) \rightarrow H o\left(\mathscr{C}_{0}\right)
$$

which is right adjoint to the inclusion $H o\left(\mathscr{C}_{0}\right) \rightarrow H o\left(\right.$ pro- $\left.\mathscr{C}_{0}\right)$. This adjointness implies that, for each $\mathrm{X}$ in $H o\left(\right.$ pro- $\left.\mathscr{C}_{0}\right)$, we have a canonical natural morphism in $H o\left(\right.$ pro- $\left.\mathscr{C}_{0}\right)$

$$
\mathbf{h}: \text { holim } \mathbf{X} \rightarrow \mathbf{X} \text {. }
$$

We shall need to assume that a Whitehead Theorem holds in $H o\left(\mathscr{C}_{0}\right)$.

Whitehead Theorem 7.7. A map $f: X \rightarrow Y$ in $H o\left(\mathscr{C}_{0}\right)$ which induces isomorphisms, $f_{\#}: \pi_{k}(X) \rightarrow \pi_{k}(Y)$ for each $k \geqq 1$, is an isomorphism in $H o\left(\mathscr{C}_{0}\right)$.

Such a theorem holds for $\mathscr{C}_{0}=\mathbf{S}_{0}$ or $\mathbf{S} p_{0}$.

The following properties of the map $h(7.6)$ are well known, and easy to deduce from the Bousfield-Kan spectral sequence $([6, \S 4.9])$, together with 7.7 . We omit the proof.

Lemma 7.8. (i) If $\mathbf{X}$ in $H o\left(\right.$ pro- $\left.\mathscr{C}_{0}\right)$ is stable in pro-Ho( $\left(\mathscr{C}_{0}\right)$, then $\mathbf{h}$ is an isomorphism in pro- $\mathrm{Ho}\left(\mathscr{C}_{0}\right)$.

(ii) If $\mathbf{X}$ in $H o\left(\right.$ pro- $\left.\mathscr{C}_{0}\right)$ is stable in $H o\left(\right.$ pro- $\left.\mathscr{C}_{0}\right)$, then $\mathbf{h}$ is an isomorphism in $H o\left(\right.$ pro- $\left.\mathscr{C}_{0}\right)$.

7.9. Proof of Theorem 2.6. To prove Theorem 2.6, we need only assume that $H o\left(\mathscr{C}_{0}\right)$ satisfies 6.2 and 7.7. This is the case for $\mathscr{C}_{0}=\mathbf{S}_{0}$ or $\mathbf{S} p_{0}$. 
If $\mathbf{X}$ is stable in $H o\left(\right.$ pro- $\left.\mathscr{C}_{0}\right)$, then trivially it is stable in pro- $H o\left(\mathscr{C}_{0}\right)$. Suppose, then that $\mathrm{X}$ in $H o\left(\right.$ pro- $\left.\mathscr{C}_{0}\right)$ is stable in pro- $H o\left(\mathscr{C}_{0}\right)$. Then $\mathrm{X}$ is strongly-movable in pro- $H o\left(\mathscr{C}_{0}\right)$ by Theorem 2.5. Also, by Lemma 7.8(i), $\mathbf{h}:$ holim $\mathbf{X} \rightarrow \mathbf{X}$ is an isomorphism in pro- $H o\left(\mathscr{C}_{0}\right)$.

By the appendix, $\mathbf{X}$ is canonically isomorphic in pro- $\mathscr{C}_{0}$ to an object indexed by $I \in C S D S$. Clearly, if $\mathbf{X}$ is stable in pro- $H o\left(\mathscr{C}_{0}\right)$, so is any object isomorphic to it. Hence, we may assume that $\mathrm{X}$ is indexed by $I \in C S D S$.

Let $\tilde{I}(\mathbf{X})$ be as in Lemma 7.3. Then, for each $\mathbf{j} \in \tilde{I}(\mathbf{X})$, the composite holim $\mathbf{X} \stackrel{\bullet}{\rightarrow} \mathbf{X} \stackrel{u_{i}}{\rightarrow} \mathbf{X}_{\mathbf{j}}$ is an isomorphism in pro- $\mathrm{Ho}\left(\mathscr{C}_{0}\right)$. Regard $\mu_{\mathrm{j}} \circ \mathrm{h}$ as a morphism in $\mathrm{Ho}$ (tow- $\left.\mathscr{C}_{0}\right)$. Then, since holim $\mathbf{X}$ is stable, it follows from [6, Corollary 5.2.13, page 178], that $\mu_{\mathrm{j}} \circ \mathrm{h}$ is an isomorphism in $H o$ (tow- $\left.\mathscr{C}_{0}\right)$, and hence in $H o\left(\right.$ pro- $\left.\mathscr{C}_{0}\right)$.

Now consider the full subcategory $\tilde{I}(\mathbf{h}) \subseteq \tilde{I}$, defined in the remarks preceding Theorem 7.1. By Remarks 7.2, $\tilde{I}(\mathbf{h})$ is the full subcategory generated by those $\mathbf{i} \in \tilde{I}$ for which $\boldsymbol{\mu}_{\mathrm{i}} \circ \mathbf{h}$ :holim $\mathbf{X} \rightarrow \mathbf{X}_{\mathrm{i}}$ is an isomorphism in $H o\left(\right.$ pro- $\left.\mathscr{C}_{0}\right)$. We have therefore shown that $\tilde{I}(\mathbf{X}) \subseteq \tilde{I}(\mathbf{h}) \subseteq \tilde{I}$. But, by Lemma 7.3, $\tilde{I}(\mathbf{X}) \subseteq \tilde{I}$ is cofinal, and hence, so it $\tilde{I}(\mathbf{h}) \subseteq \tilde{I}$. That $\mathbf{h}$ is an isomorphism in $H o\left(\right.$ pro- $\left.\mathscr{C}_{0}\right)$ now follows from Theorem 7.1. Thus, $X$ is stable in $H o\left(\right.$ pro- $\left.\mathscr{C}_{0}\right)$, and the proof of Theorem 2.6 is complete.

7.10. Proof of Proposition 2.9. By hypothesis, both $X$ and $Y$ are stable in pro- $H o\left(\mathscr{C}_{0}\right)$. Hence, by Theorem 2.6 , they are stable in $H o\left(\right.$ pro- $\left.\mathscr{C}_{0}\right)$. We then have, by the naturality of $h(7.6)$, a commutative diagram in $H o\left(\right.$ pro- $\left.\mathscr{C}_{0}\right)$

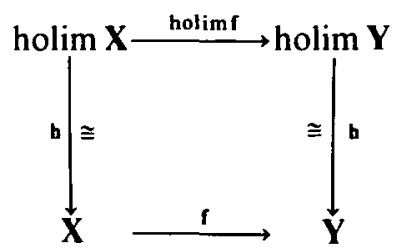

Since $\mathbf{f}$ is an isomorphism in pro- $\mathrm{Ho}\left(\mathscr{C}_{0}\right)$, it is a weak pro-homotopy equivalence (i.e., it induces isomorphisms of pro-groups on pro-homotopy groups). Hence, by [11, Theorem B], holim $\mathrm{f}$ is an isomorphism in $H o\left(\mathscr{C}_{0}\right)$, and therefore in $H o\left(\right.$ pro- $\left.\mathscr{C}_{0}\right)$. That $f$ is an isomorphism in $H o\left(\right.$ pro- $\left.\mathscr{C}_{0}\right)$ now follows from diagram (7.11).

\section{Proof of Proposition 5.1}

Recall that, if $\mathbf{X}$ is an object $\mathscr{D}^{J}$ for some category $\mathscr{D}$, and $\mathbf{X}$ is stable in pro- $\mathscr{D}$, then $\lim _{J} \mathbf{X}$ exists in $\mathscr{D}$, and the canonical morphism $h: \lim _{J} \mathbf{X} \rightarrow \mathbf{X}$ is an isomorphism in pro- $\mathscr{D}$. Thus, if $\hat{\mathbf{F}}$ is stable in pro- $\mathscr{C}^{I}$, the canonical morphism $\mathbf{h}: \lim _{\boldsymbol{J}} \hat{\mathbf{F}} \rightarrow \hat{\mathbf{F}}$ is an isomorphism in pro- $\mathscr{C}^{l}$.

Now $\lim _{J} \hat{\mathbf{F}}$ is the object of $\mathscr{C}^{I}$ given by $i \rightarrow \lim _{j \in J} F_{i j}$. Hence, by Proposition A.3, 
given $j \in J$, there is a $k \rightarrow j$ and a morphism $\theta$ in $\mathscr{C}^{I}$ such that the following diagram commutes in $\mathscr{C}^{I}$

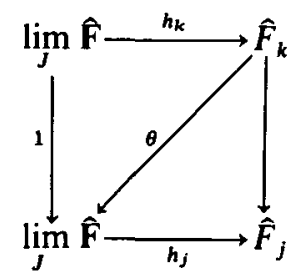

Take the colimit over $l$ of this diagram to obtain a diagram in $\mathscr{C}$

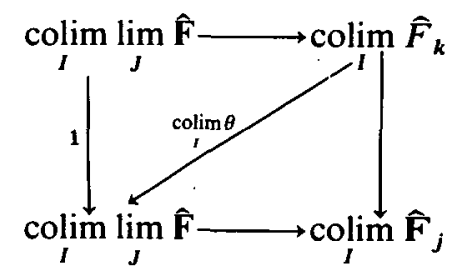

Now let $p_{j}: \lim _{J} \operatorname{colim}_{I} \hat{\mathbf{F}} \rightarrow \operatorname{colim}_{I} \hat{F}_{j}$ be the projection and define $\phi: \lim _{J} \operatorname{colim}_{I} \hat{\mathbf{F}} \rightarrow$ $\operatorname{colim}_{I} \lim _{J} \hat{F}$ by: $\phi=\left(\operatorname{colim}_{I} \theta\right) \circ p_{k}$.

We claim that $\phi$ is independent of the choice of $k \rightarrow j$ and $\theta$. Suppose that $k^{\prime} \rightarrow j^{\prime}$ and $\theta^{\prime}$ are another such choice. We must show that $\left(\operatorname{colim}_{1} \theta^{\prime}\right) \circ p_{k^{\prime}}=\left(\operatorname{colim}_{1} \theta\right) \circ p_{k}$.

Embed $k \rightarrow j$ and $k^{\prime} \rightarrow j^{\prime}$ in a diagram

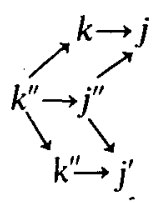

We may assume that $k^{\prime \prime}$ is chosen so that there is a $\theta^{\prime \prime}$ making the following diagram commute in $\mathscr{C}^{I}$

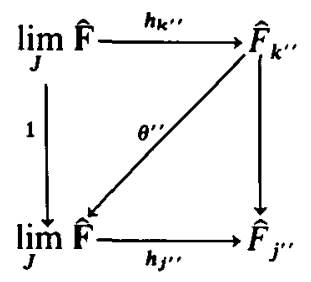

However, we do not know that $\theta \circ\left(\hat{F}_{k^{\prime \prime}} \rightarrow \hat{F}_{k}\right)=\theta^{\prime \prime}=\theta^{\prime} \circ\left(\hat{F}_{k^{\prime \prime}} \rightarrow \widehat{F}_{k^{\prime}}\right)$. We must show that $\theta^{\prime \prime}$ can be chosen so that this is the case.

By construction, both $\theta$ and $\theta^{\prime \prime}$ represent $\mathbf{h}^{-1}: \hat{\mathbf{F}} \rightarrow \lim _{J} \hat{\mathbf{F}}$ in pro- $-\mathscr{C}^{I}$. Hence, the maps

$$
\mathscr{C}^{I}\left(\hat{F}_{k} ; \lim _{J} \hat{\mathbf{F}}\right) \rightarrow \operatorname{colim}_{j \in J} \mathscr{C}^{I}\left(\hat{F}_{j} ; \lim _{J} \hat{\mathbf{F}}\right)=\operatorname{pro}^{I} \mathscr{C}^{I}\left(\hat{\mathbf{F}} ; \lim _{J} \hat{\mathbf{F}}\right)
$$


and

$$
\mathscr{C}^{I}\left(\hat{F}_{k^{\prime}} ; \lim _{J} \hat{\mathbf{F}}\right) \rightarrow \operatorname{colim}_{j \in J} \mathscr{C}^{I}\left(\hat{F}_{j} ; \lim , \hat{\mathbf{F}}\right)=\operatorname{pro}-\mathscr{C}^{I}\left(\hat{\mathbf{F}} ; \lim _{J} \hat{\mathbf{F}}\right)
$$

send $\theta$ and $\theta^{\prime \prime}$ to $\mathbf{h}^{-1}$, respectively. It follows that there is a diagram in $J, k \leftarrow l \rightarrow k^{\prime \prime}$, such that the two composites $\theta \circ\left(\hat{F}_{l} \rightarrow \hat{F}_{k}\right)$ and $\theta^{\prime \prime} \circ\left(\hat{F}_{l} \rightarrow \hat{F}_{k^{\prime \prime}}\right)$, are equal.

Similarly, we may find a diagram, $k^{\prime} \leftarrow l^{\prime} \rightarrow k^{\prime \prime}$, such that $\theta^{\prime} \circ\left(\hat{F}_{l^{\prime}} \rightarrow \hat{F}_{k^{\prime}}\right)=\theta^{\prime \prime} \circ\left(\hat{F}_{l^{\prime}} \rightarrow \hat{F}_{k^{\prime \prime}}\right)$. Now choose a diagram, $l \leftarrow l^{\prime \prime} \rightarrow l^{\prime}$. Then we have

$$
\theta \circ\left(\hat{F}_{l^{\prime \prime}} \rightarrow \hat{F}_{k}\right)=\theta^{\prime \prime} \circ\left(\hat{F}_{l^{\prime \prime}} \rightarrow \hat{F}_{k^{\prime \prime}}\right)=\theta^{\prime} \circ\left(\hat{F}_{l^{\prime \prime}} \rightarrow \hat{F}_{k^{\prime}}\right)
$$

in $\mathscr{C}^{I}$. Taking colimits over $I$ then gives

$$
\begin{aligned}
\left(\operatorname{colim}_{I} \theta\right) \circ\left(\operatorname{colim}_{I} \hat{F}_{l^{\prime \prime}} \rightarrow \operatorname{colim}_{I} \hat{F}_{k}\right) & =\left(\operatorname{colim}_{I} \theta^{\prime}\right) \circ\left(\operatorname{colim}_{I} \hat{F}_{l^{\prime \prime}} \rightarrow \operatorname{colim}_{I} \hat{F}_{k^{\prime \prime}}\right) \\
& =\left(\operatorname{colim}_{I} \theta^{\prime}\right) \circ\left(\operatorname{colim}_{I} \hat{F}_{l^{\prime \prime}} \rightarrow \operatorname{colim}_{I} \hat{F}_{k^{\prime}}\right)
\end{aligned}
$$

We also have, by definition of the maps $p_{j}$, that

$$
\left(\operatorname{colim}_{I} \hat{F}_{l^{\prime \prime}} \rightarrow \operatorname{colim}_{I} \hat{F}_{k}\right) \circ p_{l^{\prime \prime}}=p_{k}
$$

and

$$
\left(\operatorname{colim}_{1} \hat{F}_{l^{\prime \prime}} \rightarrow \operatorname{colim}_{1} \hat{F}_{k^{\prime}}\right) \circ p_{l^{\prime \prime}}=p_{k^{\prime}}
$$

Combining these with $(8.1)$ gives the result $\left(\operatorname{colim}_{I} \theta\right) \circ p_{k}=\left(\operatorname{colim}_{I} \theta^{\prime \prime}\right) \circ p_{l^{\prime \prime}}=\left(\operatorname{colim}_{I} \theta^{\prime}\right) \circ$ $p_{k^{\prime}}$, as required.

To prove the proposition, we shall show that $\phi=\left(\operatorname{colim}_{I} \theta\right) \circ p_{k}$ is the inverse of $\kappa$. Now $\kappa$ is uniquely characterized by the property that, for each $j \in J, p_{j} \circ \kappa: \operatorname{colim}_{I} \lim _{J} \hat{\mathbf{F}}$ $\rightarrow \operatorname{colim}_{I} \hat{F}_{j}$ is the colimit over $I$ of the canonical projection $h_{j}: \lim _{J} \hat{\mathbf{F}} \rightarrow \hat{F}_{j}$, in $\mathscr{C}^{I}$. We therefore have

$$
\begin{aligned}
\phi \circ \kappa & =\left(\operatorname{colim}_{I} \theta\right) \circ p_{k} \circ \kappa=\left(\operatorname{colim}_{I} \theta\right) \circ\left(\operatorname{colim}_{I} h_{k}\right) \\
& =\operatorname{colim}_{I}\left(\theta \circ h_{k}\right)=\operatorname{colim}_{I}\left(1_{\lim _{J}} \hat{f}\right)=1_{\operatorname{colim}_{I} \lim _{J} \mathrm{~F}} .
\end{aligned}
$$

Conversely, given $j \in J$, we may represent $\phi$ in the form $\phi=\left(\operatorname{colim}_{l} \theta\right) \circ p_{k}$ for some $k \rightarrow j$ and $\theta$ (since $\phi$ is independent of which representation we choose). Hence, 


$$
\begin{aligned}
p_{j} \circ \kappa \circ \phi & =p_{j} \circ \kappa \circ\left(\operatorname{colim}_{I} \theta\right) \circ p_{k} \\
& =\left(\operatorname{colim}_{I} h_{j}\right) \circ\left(\operatorname{colim}_{I} \theta\right) \circ p_{k} \\
& =\left(\operatorname{colim}_{I}\left(h_{j} \circ \theta\right)\right) \circ p_{k} \\
& =\left(\operatorname{colim}_{I} \hat{F}_{k} \rightarrow \operatorname{colim}_{I} \hat{F}_{j}\right) \circ p_{k}=p_{j} .
\end{aligned}
$$

By the universal property of limits, it now follows that $\kappa \circ \phi=$ identity on $\lim _{J}$ colim $_{I} \hat{\mathbf{F}}$. This proves the proposition.

\section{Appendix}

Let Cofilt be the category whose objects are small cofiltering (or left filtering) categories, with cofinal functors as morphisms. $I \in$ Cofilt is a strongly-directed-set if there is at most one morphism between any two objects of $I$, and if there exist morphisms $i \rightarrow j$ and $j \rightarrow i$, then $i=j$. A strongly-directed-set is cofinite if there are only finitely many morphisms out of each object. Let CSDS denote the full subcategory of Cofilt generated by the cofinite strongly-directed-sets.

Let $\mathscr{C}$ be any category. Recall [1, appendix] that a pro-object in $\mathscr{C}$ is a functor $\mathrm{X}: I \rightarrow \mathscr{C}$ for some $I \in$ Cofilt, with morphism set given by:

$$
\operatorname{pro}-\mathscr{C}(\mathbf{X} ; \mathbf{Y})=\lim _{j \in J} \operatorname{colim} \mathscr{C}\left(X_{i} ; Y_{j}\right)
$$

where $\mathrm{Y}: J \rightarrow \mathscr{C}$. With these definitions we obtain a category pro- $\mathscr{C}$, of pro-objects in $\mathscr{C}$.

Next recall $([6, \S 2],[11,1.2$ and 1.5$])$, that there is a functor $M$ :pro- $\mathscr{C} \rightarrow$ pro- $\mathscr{C}$ (the "Mardešić construction") and a natural isomorphism $\mathrm{X} \cong M \mathrm{X}$, with $M \mathrm{X}$ indexed by a cofinite strongly-directed set.

If $\phi: I \rightarrow J$ is a morphism in Cofilt, then there is a natural isomorphism in pro- $\mathscr{C}$.

$$
\mu_{\phi}: \mathbf{X} \rightarrow \phi^{*} \mathbf{X}
$$

for any pro-object $\mathbf{X}$ indexed by $J$, where $\phi^{*} \mathbf{X}=\mathbf{X} \circ \phi$ is the induced pro-object indexed by $I([1$, appendix $])$.

Recall that any morphism $\mathbf{f : X} \rightarrow \mathbf{Y}$ may be "uniformly reindexed" ([1, appendix $])$. We shall need the details of this construction. If $\mathbf{X}$ is indexed by $I$ and $\mathbf{Y}$ by $J$, we say that a morphism in $\mathscr{C}, g: X_{i} \rightarrow Y_{j}$, represents $f$ if the image of $g$ under $\mathscr{C}\left(X_{i} ; Y_{j}\right) \rightarrow$ 
$\operatorname{colim}_{i \in I} \mathscr{E}\left(X_{i} ; Y_{j}\right)$ coincides with the image of $\mathbf{f}$ under pro- $\mathscr{C}(\mathbf{X} ; \mathbf{Y})=$ $\lim _{j \in J} \operatorname{colim}_{i \in I} \mathscr{C}\left(X_{i} ; Y_{j}\right) \rightarrow \operatorname{colim}_{i \in I} \mathscr{C}\left(X_{i} ; Y_{j}\right)$. If $h: X_{i^{\prime}} \rightarrow Y_{j^{\prime}}$ also represent $f$, then a morphism: $g \rightarrow h$ is a pair of morphisms $i \rightarrow i^{\prime}$ and $j \rightarrow j^{\prime}$, such that the following square commutes

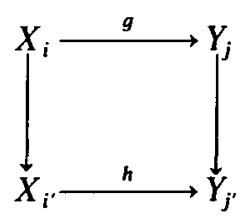

We obtain, therefore, a category $\Delta(\mathbf{f})$ whose objects are the morphisms representing $\mathbf{f}$. Define $\phi: \Delta(\mathbf{f}) \rightarrow I$ and $\psi: \Delta(\mathbf{f}) \rightarrow J$ by $\phi(g)=i$ and $\psi(g)=j$. Then it can be shown that $\Delta(\mathbf{f})$ is small and cofiltering, and $\phi$ and $\psi$ are cofinal. Now define a "uniformly indexed" morphism f: $\phi^{*} \mathbf{X} \rightarrow \psi^{*} \mathbf{Y}$, by $f_{g}=g: X_{\phi(g)} \rightarrow Y_{\psi(g)}, g \in \Delta(\mathbf{f})$. Then, by the naturality of (A.1), we have a commutative diagram in pro- $\mathscr{C}$

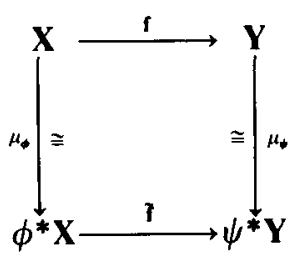

With the above machinery, we can now classify isomorphisms in pro- $\mathscr{C}$. The proposition we shall give is slightly more general than is usual (cf. [11, Proposition 1.8]; [6, Lemma 5.5.4]).

Proposition A.3. Let $\mathrm{f}: \mathrm{X} \rightarrow \mathrm{Y}$ be a morphism in pro- $\mathscr{C}$, with $\mathbf{X}$ indexed by I and $\mathbf{Y}$ by J. Then $\mathbf{f}$ is an isomorphism in pro- $\mathscr{C}$ if and only if, for each morphism $g: X_{i} \rightarrow Y_{j}$ representing $f$, there are morphisms $k \rightarrow i$ in $I$ and $l \rightarrow j$ in $J$, a morphism $h: X_{k} \rightarrow Y_{l}$ representing $f$, and a morphism $\theta: Y_{i} \rightarrow X_{i}$, such that the following diagram commutes.

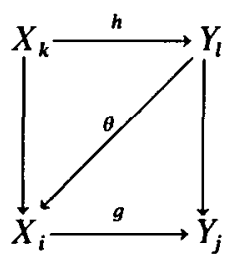

Proof. Let $\Delta(\mathbf{f}), \phi$ and $\psi$ be as described above. By (A.2), $f$ is an isomorphism if and 
only if $\mathbf{f}$ is. But $\mathbf{f}$ is a uniformly indexed morphism, and the proposition for such morphisms is well known (e.g. [11, Proposition 1.8]; [6, Lemma 5.5.4]). This latter proposition applied to $f$ translates into the given statement.

Now let $\phi: J \rightarrow I$ be any functor (not necessarily cofinal) with $I, J \in C o f i l t$, and let $\mathbf{X}$ be a pro-object indexed by $I$. We may still construct a canonical morphism $\mu_{\phi}: \mathbf{X} \rightarrow \phi^{*} \mathbf{X}$, to be the image of $1_{x}$ under the canonical map

$$
\text { pro- } \mathscr{C}(\mathbf{X} ; \mathbf{X})=\lim _{i^{\prime} \in I} \operatorname{colim} \mathscr{C}\left(X_{i} ; X_{i^{\prime}}\right) \rightarrow \lim _{j \in J} \operatorname{colim} \mathscr{C}\left(X_{i} ; X_{\phi(j)}\right)=\operatorname{pro}-\mathscr{C}\left(\mathbf{X} ; \phi^{*} \mathbf{X}\right),
$$

(though if $\phi$ is not cofinal, this map need not be an isomorphism). We wish to find conditions under which $\mu_{\phi}$ is an isomorphism.

First observe that, if $i \rightarrow \phi(j)$ is a morphism in $I$, then the induced map $X_{i} \rightarrow X_{\phi(j)}$ represents $\mu_{\phi}$. Now define a category $\Delta_{\phi}$ to have as objects the morphisms $i \rightarrow \phi_{(j)}$ in $I$, and as morphisms the commutative squares

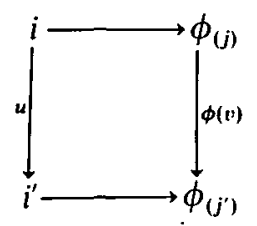

Define a functor $\rho: \Delta_{\phi} \rightarrow \Delta\left(\mu_{\phi}\right)$ by $\rho(i \rightarrow \phi(j))=\left(X_{i} \rightarrow X_{\phi(j)}\right)$ on objects, and similarly on morphisms. We then have the following lemma, the proof of which is routine and left to the reader.

Lemma A.4. $\Delta_{\phi}$ is cofiltering and $\rho$ is cofinal.

Proposition A.5. Let $\phi: J \rightarrow I$ be any functor with $I$ and $J \in$ Cofilt, and let $\mathbf{X}$ be a proobject indexed by $I$. Then the canonical morphism $\mu_{\phi}: \mathbf{X} \rightarrow \phi^{*} \mathbf{X}$, is an isomorphism in pro- $\mathscr{C}$ if and only if, for each $i \rightarrow \phi(j)$ in $I$, there are morphisms $i^{\prime} \rightarrow i$ in $I, j^{\prime} \rightarrow j$ in $J, i^{\prime} \rightarrow \phi\left(j^{\prime}\right)$ in $I$, and $\theta: X_{\phi\left(j^{\prime}\right)} \rightarrow X_{i}$ in $\mathscr{C}$, such that the following diagram commutes

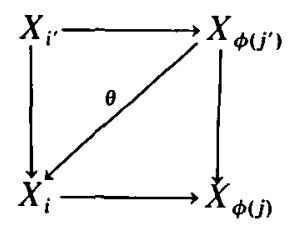

Proof. This follows immediately from Proposition A.3 and Lemma A.4.

Remark. If $\phi$ is cofinal, then the morphism $\theta$ in the above diagram can be chosen to be induced from a morphism $\phi\left(j^{\prime}\right) \rightarrow i$ in $I$.

\section{REFERENCES}

1. M. Artin and B. Mazur, Étale Homotopy, (Lecture Notes in Math., 100, Springer-Verlag, Berlin-Heidelberg-New York, 1969). 
2. K. Borsuk, A note on the theory of shape of compacta, Fund. Math. 67 (1970), 265-278.

3. J. DYDAK, On the Whitehead Theorem in pro-homotopy and on a question of Mardešic, Bull. Acad. Polon. Sci. Sér. Sci. Math. Astron. Phys. 23 (1975), 775-779.

4. D. A. Edwards and R. Geoghegan, The stability problem in shape and pro-homotopy, Trans. Amer. Math. Soc. 214 (1975), 261-277.

5. D. A. Edwards and R. Geoghegan, Stability theorems in shape and pro-homotopy, Trans. Amer. Math. Soc. 222 (1976), 389-403.

6. D. A. Edwards and H. M. Hastings, Cech and Steenrod Homotopy Theories with Applications to Geometric Topology (Lecture Notes in Math. 542, Springer-Verlag, Berlin-Heidelberg-New York, 1976).

7. S. Mardešıc, Strongly Movable Compacts and Shape Retracts (Proc. Intern. Symp. on Top. and its Appl., Budva, 1972, Savez Društava Mat. Fiz. Astron., Beograd, 1973), 163-166.

8. S. MARDEŠIC and J. SeGAL, Shape Theory: the Inverse System Approach (North-Holland, Amsterdam-New York, 1982).

9. T. Porter, Stability results for topological spaces, Math. Z. 140 (1974), 1-21.

10. D. Quillen, Homotopical Algebra (Lecture Notes in Math. 43, Springer-Verlag, BerlinHeidelberg-New York, 1967).

11. R. M. Seymour, Whitehead Theorems in pro-homotopy theory, J. London Math. Soc. 32(2) (1985), 337-356.

Department of Mathematics

University College

Gower Street

LONDON WC1E 6BT 